\title{
ANXIETY, RESILIENCE AND COPING STRATEGIES AMONG THE HEALTH CARE WORIKERS DURING COVID-19 PANDEIMIC
}

KEY WORDS: Covid-Anxiety; Resilience; coping style

\section{Varsha $\mathbf{S *}$}

\section{Dr. Smitha}

\section{Ruckmani}

\section{M.Phil.Clinical Psychology, Institute of Mental Health, Chennai, India.} *Corresponding Author

\section{Associate professor and Head of the Department, Institute of Mental Health,} Chennai, India.

Background: The covid-19 pandemic has proved to be the most difficult period of everyone's life, as it brings with it the fear of infection, fatality, physical difficulties, lack of certainty around people's life, However it is considerably challenging for the health care workers, for their nature of work makes them more vulnerable to get infected than the normal population, It is common for the health care workers to experience anxiety in the face of current pandemic. thus the present study focuses on the anxiety levels and coping strategies among health care workers during pandemic .The objective of this study is to assess levels of anxiety, resilience and ways of coping among health care workers. A total of 40 samples shall be taken for the present study, The standardized tools to be used in the study are a) Covid- 19 anxiety scale

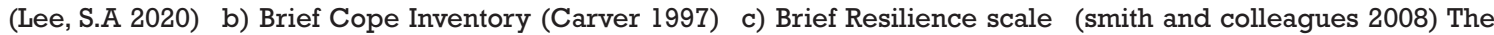
hypotheses of the current study states that 1 )There is a significant level of anxiety among health care workers 2) There is a significant relationship between the levels of anxiety and coping strategy 3) There is a significant relationship between resilience and levels of anxiety, 4) There is a significant difference between gender and level of anxiety 5) There is a significant difference between gender and coping strategy. The present study shall be insightful in terms of understanding the prevalence of anxiety among health care workers, and the role of coping strategy plays in elevating or ameliorating anxiety, It can be helpful in planning the interventions for future studies. The present study shall be helpful in understanding the level of anxiety among health care workers and what kind of coping strategy are predominantly used by the health care workers, and how adaptive/ maladaptive coping strategy influence the levels of anxiety, thus it can be helpful in planning the appropriate interventions for the target population in the future studies.

\section{INTRODUCTION:}

The covid-19 pandemic, also called as the black swan event, (Renjen, 2020) lead to an immense global unrest. The pandemic has proved to be expensive in terms of costing people's life, finance, social ties, physical health and mental health. The pandemic created a ripple effect of affecting each and every individual's life. (Nicola et al., 2020). The extent of the pandemic was such that it was declared as a global emergency by (WHO, 2020).

There were many cases reported in the city of Wuhan after the outbreak as the situation became extremely uncontrollable and the city of Wuhan reported a fatality rate of 3,869 in the month of April.

Soon after the outbreak in china, many countries begun to report cases of individuals tested positive for novel corona virus. Italy was hit with $110 \quad 574$ registered cases and $13 \quad 155$ registered deaths related to severe acute respiratory syndrome corona virus 2 (SARS-CoV-2) infection as of April 1, 2020. India reported its first confirmed case of corona on January 30 2020, in kerela, ( Perappadan 2020). The affected person had a travel history from Wuhan. On may $8^{\text {th }}$ India reported 56,342 positive cases. The news papers and magazines were filled with statistical reports of patients diagnosed with covid- 19 and the fatality rates. A nation wise lockdown was declared on march $24 / 3 / 2020$ in order to control the outspread of the virus. (Ministry of Home Affairs). As much as the physical aspects of contracting the virus, there were also reports of mental health issues among the patients diagnosed with covid-19 as they were quarantined in the hospital and there was increased anxiety, and fear of stigma after contracting the illness. (Talevi et al.,2020).

(Gupta and sahoo 2020)The front line health care workers were equally affected as much as the people who were diagnosed with covid-19. In spite of the conundrums of media reports focusing completely on novel corona virus and it fatality rate,sociological variables like job stress, fear of stigma and lack of adequate safety measures made the health care workers more vulnerable to mental health problems. which put forth the need of certified mental health professionals, to deal with the mental health crisis that came along with the pandemic.(Banerjee 2020)

Thus with front line workers, with increased exposure towards the virus, a midst the cacophony of media and reports recording massive number of cases everyday, It is not uncommon that the health care workers would have developed anxiety.

However there are also empirical evidence which suggests that health care workers have better coping mechanism in dealing with a triggering situation than the normal population. owing to their ability to discern the credibility of an information, experiential learning from previous medical emergencies.Thus medical health care worker can handle mental health issues associated with a pandemic effectively than non medical health care worker(Tan et al.2020).

There are ample of studies, which articulates about the resilience among health care workers, A pandemic like covid19 , which demands hardiness as a cardinal trait to deal with a situation, most of the studies have reported that the health care workers have higher resilience , (Mosheva et al., 2020). However most of the studies have focused on health care workers and the mental health outcomes in general. The present study bridges the gap by understanding the mental health outcomes of health care workers who are working in the covid-19 pandemic.

\section{Literature Review}

The covid -19 pandemic has been found to be very much detrimental to human lives owing to the fatality rate and physical illness that came along with it. Though the nature of the pandemic seem to affect the physical health, it was during the pandemic that there were upsurge of mental health issues among the public and also the front line workers. The uncertainty and lack of clear information about the nature of the illness instigated anxiety among the public, there were wide spread of mental health issues such as depression, stress and anxiety. Many studies reported that the pandemic has 
proved to be as much as a mental health concern. (Petzold et al.,2020). studied the risk, resilience, psychological distress and anxiety at the beginning of the covid-19 in Germany. The results of the study indicated that $50 \%$ of the samples expressed anxiety and psychological distress due to covid-19 pandemic. However psychological factors such as resilience, coping style such as taking social support, healthy lifestyle played as protective factors.

However there are substantiate amount of research evidence that confirms to the view that health care workers are most prone to develop anxiety, however the personal resources such as coping style, resilience play as protective factors by making them less vulnerable to develop anxiety ( Tan et al.,2020). studied the psychological impact of the pandemic on health care workers working in Singapore, From the findings of DASS-21 and IES-R scores , the mean scores of the health care workers were lower than the previous pandemic such as SARS. A very interesting finding from the study, is that the health care workers who are in front line duty experience less anxiety and depression than the non medical para medical professionals, who do not have as much expertise in dealing with an outbreak, because of the lack of their experience in being on the front line duty. This is seen as an important factor for them to develop uncertainty and anxiety than the experienced medical professionals. The study substantiated the above mentioned hypotheses with a recent covid -19 study, which explored the difference in coping pattern between medical health care workers and non medical health care workers. It was seen that front line nurses had lesser vicarious trauma scores than the non front line nurses. Thus the study suggests appropriate interventions for non medical health care workers in managing their mental health issues such as depression, stress and anxiety due to the pandemic.

The covid- 19 pandemic was found to be even more detrimental for the physicians and they had more stress and depression, and only few percentage of samples had good resilience, (Khalil et al.,2020) studied the Egyptian physicians mental health on the dimensions of stress, depression and coping styles. It was found that $63 \%$ of the samples suffered from severe or extremely severe depression. From the findings based on BRCS, it was found that only $17.1 \%$ of the samples had high resilience and that of the resilience scores negatively correlated with scores of DASS on anxiety, depression and stress.

Most of the literature summarizes the protective role of coping and resilience in managing the symptoms of anxiety, it is seen that there is an inverse relationship between resilience, coping style and anxiety.

Coping is one of the many factors that play a protective role in making the individual less vulnerable to develop anxiety. Coping is negatively associated with anxiety (Zhu et al., 2020) studied the prevalence of depression and anxiety symptoms among front line medical stuffs working against covid-19. The findings of the study suggested that the prevalence rate of depression and anxiety among the front line staffs was $11.4 \%$ and $45.6 \%$, history of depression or anxiety proved to be a risk factor in developing mental health issue due to the pandemic, of which an interesting pointer in the study was that, the coping style of physicians was negatively associated with anxiety, which indicates that coping style does play a critical role in the management of anxiety.

\section{Need and Rationale of the study}

Though there are many studies, that have been carried out to explore the impact of mental health issue due to the pandemic, the number of studies that focus on the protective factors against the mental health issue due to the pandemic are limited. In order to bridge the lacunae the present study attempts to understand the relationship between anxiety, coping style and resilience. To understand if there is a relationship between these variables, so as to understand the importance of protective factors and there by incorporate in future implications of framing appropriate intervention for the target population.

\section{METHOD}

\section{Research Design:}

The Ex post-facto research design was adopted for the study.

\section{Aim:}

The study aims to find the relationship between anxiety, coping style and resilience among health care workers.

\section{Objective}

To find out the relationship between anxiety and coping style among health care workers.

To find out the relationship between anxiety and resilience among health care workers.

To find out the relationship between resilience and coping style among health care workers.

\section{Hypothesis}

There is a significant relationship between anxiety and coping style among health care workers.

There is a significant relationship anxiety and resilience among health care workers.

There is a significant relationship between coping style and resilience among health care workers.

\section{Sample Characteristics:}

The socio- demographic details of the participants are presented in the table 1. participants were health care workers ( doctors, nurse, psychologists, physiotherapist, radiographer's, and social workers who work in hospital setting or health care setting during the pandemic). Participants were above 18 years of age and the samples were selected from different hospitals in Tamilnadu.

Table 1. Socio-Demographic Details Of The Participants

\begin{tabular}{|l|l|l|l|}
\hline S.no & Variables & & Frequency \\
\hline 1 & Gender & Male & 13 \\
& & Female & 53 \\
\hline 2. & Marital Status & Married & 27 \\
& & Unmarried & 39 \\
\hline 3. & Occupation & Doctor & 10 \\
& & Nurse & 11 \\
& & Clinical- Psychologist & 13 \\
& & Clinical-Psychology & 25 \\
& & Trainees & 7 \\
\hline 4 & Shysical & Present & 1 \\
& Ailment & Absent & 65 \\
\hline 5 & Tested Positive & Positive & 9 \\
& for Covid-19 & Negative & 57 \\
\hline
\end{tabular}

\section{Sample Selection:}

The sampling method used to select the sample was purposive sampling.

\section{Inclusion Criteria}

Samples who work in hospitals or health care setting during the covid-19 pandemic ( doctors, nurse, psychologist, radiographers and social workers )

Samples who are willing to participate in the study and the participants who have signed the informed consent.

\section{Exclusion Criteria}

Samples who have different work designation than the prerequisite 
Samples who do not work in the hospital but fulfill the criteria in terms work designation.

Samples Who Have Psychiatric OrWith Co Morbid Illness

\section{Tools Used}

1. Informed consent form. An informed consent sheet providing the aim of the study, possible effects, researcher's information was given. The participants were asked to click yes after reading the consent form of the google sheet.

2. Socio- demographic information such as age, education, marital status was obtained from the samples

3. Covid-19 anxiety scale. To identify individuals who have abnormal levels of anxiety over covid-19. The scale was developed by lee et al (2020).The scale has five items which assess the severity of anxiety over covid-19. It was first analyzed among 775 adults in the united states. scores range from 5 to 25. of which 9 is the cut off score for discriminating non anxiety and dysfunctional anxiety.Dysfunctional anxiety refers to a disproportionate state of anxiety, It defined as persistent or uncontrollable fear that interferes with daily life and causes disruptions to behaviour and psychological wellbeing (Lee et al., 2020).

The scale has excellent predictive validity as substantiated by positive association between disability and distress ( Lee et al. 2020) and outstanding reliability of 0.93 (Lee et al.2020) and cronbach alpha of 0.87 .

4. Brief cope scale. Brief cope is a self report scale which consists of 28 items and it measures the adaptive and maladaptive ways of coping styles. The item consists of $14 \mathrm{sub}$ scales and the internal consistencies were good for problem focused,and dysfunctional sub scales. The scale shows excellent concurrent and convergent validity.

5. Brief resilience rating scale. To assess the health care worker's ability to bounce back from stressful events - Brief resilience scale was used. It was developed by smith et al (2008).The samples indicated their responses on a 5 point likert rating scale of which 0 is(does not describes me at all) to 5 (describes me very well). The mean scale scores were categorized into three groups: low resilience (1.00-2.99), moderate resilience (3.00-4.30), and high resilience (4.31-5.00) Smith et al., (2008). The Scale has shown predictive validity by its association with work performance, health and well being (Foster, Roche, Giandinoto, \& Furness, 2020 ), and a reliability, with an internal consistency of 0.91 in previous research (Smith et al., 2008) and a Cronbach's alpha of 0.84 .

\section{Procedure:}

The purpose of the study was explained. Informed consent was received from the samples who volunteered to take part in the study. The data collection was carried out using google forms with adequate password encryption. The editing of other sources option was turned off. Only the researcher of the current study had access to the response sheet, ensuring safety of the data. The google forms had, informed consent, demographic sheet, covid-19 anxiety scale, brief cope and brief resilience scale. All the items in the forms were marked required, except phone number owing to privacy. The google forms were sent to an official group which consists of doctors, nurses, psychologists and social workers who are posted for covid duty. Some forms were sent separately to health care workers who work in different hospitals in Tamilnadu for covid duty. The data obtained were entered in the google excel sheet and analyzed using spss.

\section{Data Analysis.}

Pearson's coefficient of correlation was obtained between three variables Ethical considerations.

\section{Ethical Procedure:}

The study followed APA format for ethical consideration.

\section{Risks Involved:}

The participants were informed of the possible risk of emotional disturbances during or post the participation in the study.

\section{Consent Form :}

The participants were given consent form, which includes details about the study and the participants willingness to participate in the study.

\section{Harming:}

No participant was harmed during the study

\section{RESULTS AND DISCUSSION}

This section contains the important tables and significant results that were obtained after the analysis of the data. The discussion section explains and justifies the results that have been obtained.

Table 2 Descriptive Statistics Of The Variables.

\begin{tabular}{|l|l|l|}
\hline & Mean & Standard Deviation \\
\hline Covid-19 anxiety & 0.80 & 1.08 \\
\hline Coping style & & \\
Approach style & 29.03 & 9.14 \\
Avoidance style & 18.63 & 6.40 \\
\hline Resilience & 3.35 & 0.70 \\
\hline
\end{tabular}

Table 2 shows the descriptive statistics for the data that were analyzed. As seen from the table a mean of 0.80 was obtained for covid-anxiety and a mean of 29.03 was obtained for approach coping style and a mean of 18.63 was obtained for avoidance coping style. A mean of 3.35 was obtained for resilience. The data result suggests that despite having low levels of anxiety, there is high level of approach style of coping and low level of avoidance style of coping with normal levels of resilience.

Table 3 Pearson's Correlation Coefficient Between The Variables Of Coping Style And Covid Anxiety

\begin{tabular}{|l|l|l|}
\hline & Avoidance coping style & Approach coping style \\
\hline Anxiety & $0.51 * *$ & 0.09 \\
\hline$* *$ correlation is significant at 0.01 level
\end{tabular}

Table 3 shows the Pearson's correlation coefficient values that were obtained between covid- anxiety and coping style. There are two types of coping styles which are mentioned in the table 3. A correlation coefficient of 0.51 which is significant at 0.01 level, has led to the acceptance of alternate hypotheses. It indicates there is a positive relationship between anxiety and avoidance coping style, in other words if the avoidance coping style increases, there is also an increase in the anxiety. From the table 3, we can also see the correlation coefficient of anxiety and approach coping style, it is seen that there is no significant relationship between anxiety and approach coping style.The results of the current study is substantiated by (zhu et al.,2020) the study focused on coping style and relationship between social support and covid anxiety among chinese medical staff, it was seen that social support was negatively related to anxiety and positive and negative coping had effects on social support and anxiety.

Table 4 Pearson's Correlation Coefficient Between The Variables Of Covid-Anxiety And Resilience

\begin{tabular}{|l|l|}
\hline & Resilience \\
\hline Anxiety & -0.17 \\
\hline
\end{tabular}

Table 4 shows the pearson's correlation coefficient values that were obtained between covid anxiety and resilience. A correlation coefficient of -0.17 indicates that there is no significant relationship between resilience and anxiety. The results of the study is substantiated with the findings of the study conducted by (Mosheva et al., 2020). Physicians reported high anxiety and it was found that there was an inverse relationship between resilience and covid- anxiety. 
Table 5 Pearson's Correlation Coefficient Between The Variables For Coping Style And Resilience

\begin{tabular}{|l|l|l|}
\hline & $\begin{array}{l}\text { Avoidance coping } \\
\text { style }\end{array}$ & $\begin{array}{l}\text { Approach coping } \\
\text { style }\end{array}$ \\
\hline Resilience & $-0.26 * *$ & 0.09 \\
\hline
\end{tabular}

$* *$ Correlation is significant at 0.05 level

Table 5 shows the pearson's correlation coefficient values that were obtained between resilience and coping style. It is seen that a coefficient correlation of -0.26 which is significant at 0.05 level, indicates that there is a significant negative relationship between resilience and avoidance coping style. In other words it means that when the avoidance coping increases, resilience decreases.

In the table 5 we can also see the relationship between resilience and approach coping style, a correlation coefficient value of 0.09 indicates that there is no significant relationship between resilience and approach coping style.( Labrague et al,.2020) studied the influence of resilience, personal factors and social support in covid anxiety and it was found that,nurses were found to have prevalent covid-19 anxiety and social support, personal resilience and resilience were predictive of covid-19 anxiety which was analyzed through regression analysis.

\section{Summary}

Anxiety is one of the common outcome of pandemic and based on the findings of many empirical studies, health care workers are more vulnerable to develop mental health issue during the time of pandemic, due to the exposure towards the virus, fear of infecting the loved ones or lack of certainty around the course of the pandemic. Thus it is not uncommon for them to develop mental health issues. However few empirical evidence also suggests that front line medical health workers experience lesser mental health problems than the non medical health care professional, as they are adequately equipped with information and their previous experiential learning in dealing with hazardous medical emergency cases. The studies focus on the protective role of personal factors such as resilience, personality disposition and coping style an $+88 \mathrm{~d}$ social factors such as social support and organizational support in making the individual less vulnerable to develop mental health issues. Thus the present study focused on the relationship between anxiety, coping style and resilience among health care workers. Data was collected using the covid-19 anxiety scale. Brief resilience scale, brief cope scale from a sample of 66 health care workers. Statistical analysis was conducted using Pearson's coefficient of correlation and T- test. Results indicated that people have lower level of avoidance coping and lower level of anxiety and people with lower level of avoidance coping had normal level of resilience.

\section{CONCLUSION:}

The results of the study showed that there is a positive relationship between avoidance coping and anxiety. In other words, if avoidance coping decreases anxiety level is decreased. There is a negative relationship between avoidance coping and resilience, in other words if the avoidance coping decreases the levels of resilience is increased.

\section{Limitation And Implications:}

The limitation of the study is a small sample size due to which the results cannot be generalized. The study was conducted using google forms, due to the present pandemic situation, thus the lack of direct contact with the samples which compromises on the transparency of the other extraneous factors such as noisy environment, or distraction while filling the form , network connectivity problems etc.. that could have potentially influenced the study tends to remain unknown. For further studies, a larger sample size which focuses on intervention based experimental approaches to understand the effectiveness of an intervention in managing the mental health issues of health care workers can be of significance. The future studies can also understand the difference in mental health outcomes between front line health care medical workers and non medical health workers to understand the difference in dealing with a medical emergency situations and to design an appropriate intervention to address the concerns and thereby understand the effectiveness of the intervention is needed.

\section{REFERENCES}

1. Allyson dantas silva, w., rayane sampio brito, t., \& roberto perira, c. (2020, November). COVID-19 anxiety scale (CAS): Development and psychometric properties. current psychology, 39(6). doi:https://link.springer. com/article/10.1007/s12144-020-01195-0

2. Banarjee, D. (2020, March). The COVID-19 outbreak: Crucial role the psychiatrists can play. BMC Public Emergency Collection. doi:10.1016/j.ajp.2020.102014

3. Boccia, S., Ricciardi, w., \& Loannidis, J. (2020, April 7). What Other Countries Can Learn From Italy During the COVID-19 Pandemic. JAMA Internal Medicine, 180(7),927-928. doi:10.1001/jamainternmed.2020.1447

4. Circulars for Covid-19 (n.d.). In Ministry of Human Affairs. Retrieved from https://www.mha.gov.in/notifications/circulars-covid-19

5. Guptha, S., \& Sahoo, S. (2020, August). Pandemic and mental health of the front-line healthcare workers: a review and implications in the Indian context amidst COVID-19. BMC Public Emergency Collection, 33(5). doi: 10.1136/gpsych-2020-100284

6. Khalaf, O.O., Khalil, M.A. \& Abdelmaksoud, R. Coping with depression and anxiety in Egyptian physicians during COVID-19 pandemic. Middle East Curr Psychiatry 27, 63 (2020). https://doi.org/10.1186/s43045-020-00070-9

7. Lai J, Ma S, Wang Y, et al. Factors Associated With Mental Health Outcomes Among Health Care Workers Exposed to Coronavirus Disease 2019. JAMA Netw Open. 2020;3(3):e203976. doi:10.1001/jamanetworkopen.2020.3976

8. Mosheva, M., Palmor, N., Ilen, s., Matalon, N., Pessach, I. M., Afek, A., ... Ziv, A. (2020, October). Anxiety, pandemic related stress and resilience among physicians during the COVID 19 pandemic. Depression and anxiety during the covid-19 pandemic and other trauma, 37(10), 965-971. doi: https://doi.org/10.1002/da.23085

9. Nicola, M., Alsafi, Z., Sohrabhi, C., Kerwan, A., Al- Jabir, A., Losifidis, C., .. Agha, R. The socio-economic implications of the coronavirus pandemic (COVID-19): A review. Elseiver's Public Health Emergency Collection, 78, 185193. doi:10.1016/j.ijsu.2020.04.018

10. Perappadan, B. (2020, January 30). India's first coronavirus infection confirmed in Kerala . In THE HINDU. Retrieved from https://www.thehindu. $\mathrm{com} /$ news/national/indias-first-coronavirus-infection-confirmed-inkerala/article30691004.ece

11. Petzold, M., Bendau, A., Plag, J., Pyrkosh, L., Maricic, L., Betzler, F., ... Strohle, A. (2020, July 7). Risk, resilience, psychological distress, and anxiety at the beginning of the COVID 19 pandemic in Germany. Brain and Behavior, 10(9). doi:https://doi.org/10.1002/brb3.1745

12. Renjen, P. (2020, March 16). The heart of resilient leadership: Responding to COVID-19 . In Deloitte insights. Retrieved from https://www2.deloitte.com/ global/en/insights/economy/covid-19/heart-of-resilient-leadershipresponding-to-covid-19.html

13. Sahoo, S., mehra, A., Suri, V., Malhotra, P., Yaddanapudi, L., Puri, G., ... Grover, S. (2020, May 30). Lived experiences of the corona survivors (patients admitted in COVID wards): A narrative real-life documented summaries of internalized guilt, shame, stigma, anger. Elseiver's Public Health Emergency Collection.doi:10.1016/j.ajp.2020.102187

14. Smith, B. W., Dalen, J., Wiggins, K., Tooley, E., Christopher, P., \& Bernard, J. (2008). The brief resilience scale: assessing the ability to bounce back. International journal of behavioral medicine, 15(3), 194-200.

15. Talevi, D., Socci, V., Carai, M., Carnaghi, G., Faleri, S., Trebbi, E., ... Pacitti, F. Mental health outcomes of the CoViD-19 pandemic. rivista di psichiatria, 55. doi: 10.1708/3382.33569

16. Tan, B., Chew, N., Lee, G., Jing, M., Goh, Y., Leo, L., ... Sharma, V. K. (2020, August 18). Psychological Impact of the COVID-19 Pandemic on Health Care Workers in Singapore. Annals of internal medicine. doi:https://doi.org/10. 7326/M20-1083

17. Validity and Reliability of the Brief COPE in Carers of People With Dementia. (2008, November). The Journal of Nervous and Mental disease, 196(11), 838846.

18. Wuhan, once China's coronavirus epicentre raises death toll by $50 \%(2020$, April 17). In WION. Retrieved from https://www.wionews.com/ world/wuhan-once-chinas-coronavirus-epicentre-raises-death-toll-by-50293040

19. Zhu, J., Sun, L., Zhang, L., Wang, H., Fan, A., Yang, B., ... Xiao, S. (2020, April 29). Prevalence and Influencing Factors of Anxiety and Depression Symptoms in the First-Line Medical Staff Fighting Against COVID-19 in Gansu. Front Psychiatry, 1 1(386).doi: 10.3389/fpsyt.2020.00386. 